\title{
Andreev Reflection in Transport through a Quantum Dot Coupled to Ferromagnetic and Superconducting Electrodes
}

\author{
K. BOCIAN ${ }^{a}$, W. RUdzińSKI ${ }^{a}$ AND J. BARNAS $\hat{S}^{a, b}$ \\ ${ }^{a}$ Department of Physics, Adam Mickiewicz University, Umultowska 85, 61-614 Poznań, Poland \\ ${ }^{b}$ Institute of Molecular Physics, Polish Academy of Sciences, M. Smoluchowskiego 17, 60-179 Poznań, Poland
}

\begin{abstract}
Spin-dependent tunneling through a quantum dot coupled to one ferromagnetic and one superconducting electrodes is studied theoretically in the Andreev reflection regime. Electrical conductance is calculated in terms of the nonequilibrium Green function technique. Effects due to the Zeeman splitting of the dot level are analyzed in both linear and nonlinear transport regimes, and are compared with the corresponding effects due to coherent intradot spin rotation.
\end{abstract}

PACS: 72.25.Mk, 73.63.Kv, 73.23.Hk

\section{Introduction}

Transport through hybrid structures consisting of quantum dots, nanoparticles, carbon nanotubes or other molecules attached via tunnel barriers to external nonmagnetic, ferromagnetic or superconducting leads have been the subject of extensive studies in recent years. In particular, some efforts have been focused on the effects due to competition of superconducting pairing, Coulomb blockade, spin-flip scattering, and interaction with boson field in electronic transport through a system consisting of ferromagnet (F), quantum dot (QD) and superconductor (S) [1-4]. It has been shown that the linear conductance due to the Andreev reflection (AR) allows to determine spin polarization at the Fermi level of an external ferromagnetic metallic electrode attached to a quantum dot [1]. The Andreev reflection in transport through a QD coupled to two ferromagnetic electrodes and a superconductor revealed some interesting properties associated with the matching condition for maximum $\left(4 e^{2} / h\right)$ linear conductance and the magnetoresistance effect. It has been also predicted that for strong enough spin-flip interaction in the dot, the interplay of spin properties of the AR processes and spin dependent electronic transport leads to a double-peak structure in the linear conductance [3]. Moreover, it has been shown that when the electron-phonon interactions in the dot are taken into account, new peaks appear on both sides of the main AR conductance peak [4].

The main objective of this paper is to extend previous studies of the hybrid F-QD-S systems [3] - where the effect of intradot spin-flip processes on the AR linear conductance was examined in detail - by considering the Andreev reflection phenomenon in the context of the interplay between tunneling processes, intradot spin-flip transitions and Zeeman spin-splitting of the discrete energy level of the dot. Moreover, both linear as well as nonlinear transport regimes are considered. In order to calculate the current-voltage characteristics we employ the nonequilibrium Green function technique, and use the equation of motion method. The assumption of vanishing Coulomb correlations in the dot allowed to find exact solutions for AR current and differential conductance.

\section{Model and method}

We consider a single-level QD coupled via tunnel barriers to one ferromagnetic and one superconducting leads. The whole system can be described by Hamiltonian of the general form

$$
H=H_{\mathrm{f}}+H_{\mathrm{s}}+H_{\mathrm{d}}+H_{\mathrm{t}} .
$$

The term $H_{\mathrm{f}}$ describes the ferromagnetic electrode in the non-interacting quasi-particle approximation, $H_{\mathrm{f}}=$ $\sum_{k, \sigma} \varepsilon_{k \sigma} f_{k \sigma}^{+} f_{k \sigma}$, where $\varepsilon_{k \sigma}$ is the single-electron energy for wave vector $k$ and spin $\sigma(\sigma=\uparrow, \downarrow)$ in the ferromagnetic electrode, whereas $f_{k \sigma}^{+}$and $f_{k \sigma}$ are the corresponding creation and annihilation operators. The second term is the BCS Hamiltonian of the superconducting lead, $H_{\mathrm{s}}=\sum_{p, \sigma} \varepsilon_{p} s_{p \sigma}^{+} s_{p \sigma}+\sum_{p}\left(\Delta^{*} s_{p \uparrow}^{+} s_{-p \downarrow}^{+}+\Delta s_{-p \downarrow} s_{p \uparrow}\right)$, with $\varepsilon_{p}$ denoting the single-particle spectrum in the corresponding normal-metal phase, and $\Delta$ being the parameter describing the superconducting energy gap. The quantum dot is described by the term $H_{\mathrm{d}}=\sum_{\sigma}\left(\varepsilon_{d}+\right.$ $\hat{\sigma} B) d_{\sigma}^{+} d_{\sigma}+R\left(d_{\uparrow}^{+} d_{\downarrow}+d_{\downarrow}^{+} d_{\uparrow}\right)$, where $\varepsilon_{d}$ denotes the energy of the discrete level, $d_{\sigma}^{+}$and $d_{\sigma}$ are the relevant creation and annihilation operators, respectively, $B$ stands for magnetic field (measured in energy units) along the quantization axis imposed by magnetization of the fer- 
romagnetic lead, $\hat{\sigma}=1$ for $\sigma=\uparrow$ and $\hat{\sigma}=-1$ for $\sigma=\downarrow$, while $R$ is the spin-flip coupling parameter (e.g. due to magnetic field normal to the quantization axis). Finally, the tunnelling term in Eq. (1) takes the form $H_{\mathrm{t}}=\sum_{k, \sigma} T_{k \sigma}^{f} f_{k \sigma}^{+} d_{\sigma}+\sum_{p, \sigma} T_{p \sigma}^{s} s_{p \sigma}^{+} d_{\sigma}+$ H.c., where $T_{k \sigma}^{f}$ $\left(T_{p \sigma}^{s}\right)$ is the tunneling amplitude between the ferromagnetic (superconducting) lead and the QD, and H.c. stands for the Hermitian conjugate terms.

Making use of the nonequilibrium Green-function technique in the $4 \times 4$ Nambu representation [5], one can calculate the current due to the Andreev reflection from the formula $[3,5]$ :

$$
\begin{aligned}
J & =\frac{e}{h} \int \mathrm{d} \omega\left[f_{l}(\omega-e V)-f_{l}(\omega+e V)\right] \\
& \times \sum_{i=1,3}^{j=2,4} G_{i j}^{\mathrm{r}}\left(\boldsymbol{\Gamma}_{\mathrm{f}} \boldsymbol{G}^{\mathrm{a}} \boldsymbol{\Gamma}_{\mathrm{f}}\right)_{j i},
\end{aligned}
$$

where $f_{l}(\omega)$ stands for the Fermi-Dirac distribution function in the left (ferromagnetic) lead, and $G^{\mathrm{r}(\mathrm{a})}$ is the retarded (advanced) Green function of the QD. Apart from this, $\boldsymbol{\Gamma}_{\mathrm{f}}$ in Eq. (2) is the coupling matrix between the QD and ferromagnetic lead, which is determined by the spin-up and spin-down coupling strengths defined as $\Gamma_{\mathrm{f} \uparrow(\downarrow)}=\Gamma_{\mathrm{f} 0}(1 \pm P)$, where $P$ is the corresponding spin polarization factor and $\Gamma_{\mathrm{fo}}$ is a coupling constant. Both $\boldsymbol{\Gamma}_{\mathrm{f}}$ and $\boldsymbol{G}^{\mathrm{r}(\mathrm{a})}$ are matrices in the Nambu space.

With these definitions, the matching condition of the Fermi velocity can be written as $\Gamma_{\mathrm{f} \uparrow} \Gamma_{\mathrm{f} \downarrow}=\Gamma_{\mathrm{s} 0}^{2}$. Here, $\Gamma_{\mathrm{s} 0}$ describes the tunneling coupling strength between the QD and the superconducting lead.

\section{Numerical results}

In the following we shall discuss features of the AR conductance at zero temperature, $T=0$, for the hybrid F-QD-S system in both linear and nonlinear response regimes. Energy is measured from the Fermi level at equilibrium, and the energy gap of the superconducting lead is taken as the energy unit. The spin polarization $P$ is assumed as $P=0.3$.

In Fig. 1 we show the linear AR conductance versus energy level $\varepsilon_{d}$ of the QD, calculated for a fixed $\Gamma_{\mathrm{s} 0}$, $\Gamma_{\mathrm{s} 0}=0.1$, and for indicated values of the coupling strength $\Gamma_{\mathrm{f} 0}$ and selected values of the external magnetic field $B$. To emphasize the features due to the Zeeman splitting, the spin-flip processes in the dot have been excluded in Fig. 1 assuming $R=0$. Let us consider first the case of $B=0$, where the AR conductance exhibits one resonance peak at $\varepsilon_{d}=0$. As one can see in the upper part in Fig. 1, the conductance is relatively small for $\Gamma_{\mathrm{f} 0}<\Gamma_{\mathrm{s} 0}$. For weak enough magnetic fields one still observes one resonance peak. However, its height increases with increasing magnitude of $B$. The conductance reaches the maximum value of $4 e^{2} / h$ at a certain magnetic field, and the peak becomes asymmetrical with respect to sign reversal of $\varepsilon_{d}$. Further enlargement of magnetic field results in a splitting of the conductance

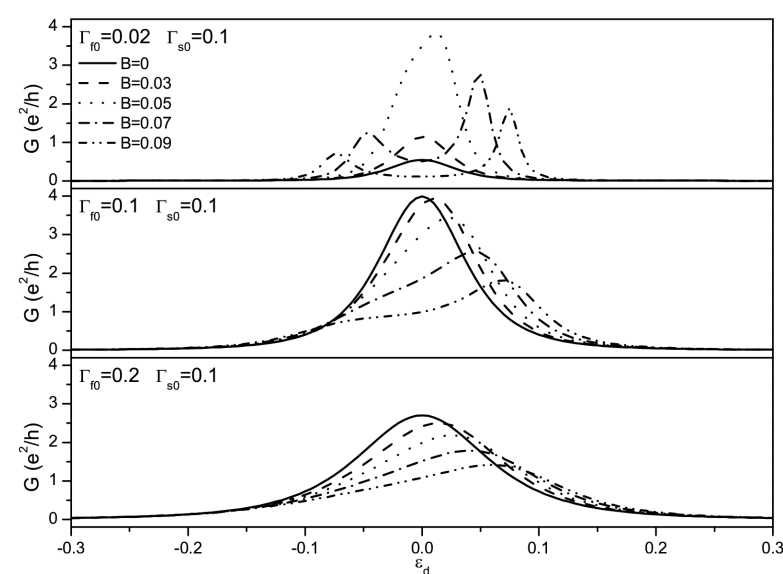

Fig. 1. AR linear conductance versus energy of the quantum dot discrete level, $\varepsilon_{d}$, calculated for indicated values of the magnetic field $B$. The other parameters are: $T=0, P=0.3, R=0, \Gamma_{\mathrm{s} 0}=0.1$, and $\Gamma_{\mathrm{f} 0}=0.02$ (upper part), $\Gamma_{\mathrm{fo}}=0.1$ (middle part), and $\Gamma_{\mathrm{f} 0}=0.2$ (bottom part)

peak into two distinct asymmetrical peaks located at the distance of $2 B$ from each other. The conductance maxima become then reduced. This behavior may be explained as follows. When the external magnetic field lifts the spin degeneracy of the dot level, the transition probabilities relevant for the AR current become different for the spin majority and spin minority carriers, which leads to the asymmetry of resonance peaks. For weak magnetic fields, the interplay of the Zeeman effect and the dot level broadening described by $\Gamma_{\mathrm{f} 0}$ leads to partial overlap of the dot spin channels and thus the AR conductance displays a single asymmetrical peak. By contrast, when the spin-flip processes are taken in to account instead of magnetic field, $R>0$ and $B=0$, then the AR resonance peaks are always symmetrical (not shown). This is in agreement with Fig. 2 of Ref. [3], and is a consequence of the fact that the dot states taking part in transport are then superpositions of spin-up and spin-down states.

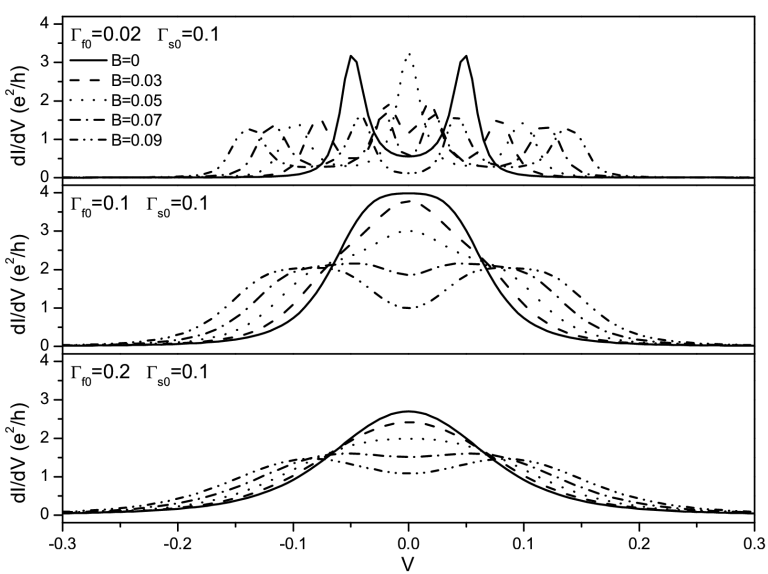

Fig. 2. Differential conductance versus bias voltage for the case of $\varepsilon_{d}=0$. The other parameters are as in Fig. 1 . 
When the coupling strength $\Gamma_{\mathrm{f} 0}$ increases, then for the specific case of $\Gamma_{\mathrm{f} 0}=\Gamma_{\mathrm{s} 0}$ one observes perfect AR tunneling processes at $\varepsilon_{d}=0$, as shown by the solid line for $B=0$ in the middle part in Fig. 1. Further increase of the parameter $\Gamma_{\mathrm{fo}}$ (see the bottom part in Fig. 1) reduces the Andreev reflection and consequently the height of the AR resonance peak is suppressed. By applying a magnetic field, one still may observe the splitting of the AR conductance at the resonance. However, for $\Gamma_{\mathrm{f} 0}>\Gamma_{\mathrm{s} 0}$ the overlap of the broadening of the spin-split dot level seems to overwhelm entirely the Zeeman effect for the assumed parameters.

The current-voltage characteristics for the nonequilibrium situation, calculated for selected values of the magnetic field $B$, are shown in Fig. 2. Consider first the upper part, where the case of $\Gamma_{\mathrm{f} 0}<\Gamma_{\mathrm{s} 0}$ is presented. As shown by the solid line, the Andreev reflection for $B=0$ and for $\varepsilon_{d}=0$ leads to renormalization of the dot energy level for both bias voltage polarizations, so that the resonance conductance peaks occur at $\pm \Gamma_{\mathrm{s} 0} / 2$. The left and right conductance peaks for $B=0$ correspond to the hole and electron Andreev transmission, respectively, and as stated earlier in [6], the particle-hole splitting is controlled by $\Gamma_{\mathrm{s} 0}$.

For a nonzero magnetic field, each resonance peak splits into two peaks separated by the distance equal to $2 B$. As a result, a large AR conductance peak appears at $V=0$ for the specific case of $B=\Gamma_{\mathrm{s} 0} / 2$. Consequently, the differential conductance at zero bias increases, exceeding significantly the value observed for $B=0$. The conductance in the middle part indicates that the effects of the dot level renormalization become diminished with increasing $\Gamma_{\mathrm{f} 0}$, and thus at the resonance a single broad peak appears. Similarly as in the linear case, perfect AR transmission through the $\mathrm{F}-\mathrm{QD}-\mathrm{S}$ system appears for $\Gamma_{\mathrm{f} 0}=\Gamma_{\mathrm{s} 0}$, i.e. the conductance reaches then maximum value equal to $4 e^{2} / h$ at the resonance. On the other hand, the splitting of the conductance peak may still be observed for large enough values of the magnetic field $B$. When $\Gamma_{\mathrm{f} 0}>\Gamma_{\mathrm{s} 0}$ (bottom part in Fig. 2), the conductance behavior is qualitatively similar to the one displayed in the middle part in Fig. 1. However stronger coupling to the F-lead diminishes the AR processes and the conductance is reduced.

Above, we have presented in detail nonlinear conductance for nonzero magnetic field and in the absence of spin-flip rotation in the dot. However, taking into account spin-flip processes $(R>0)$ and assuming zero field, $B=0$, one finds the nonequilibrium differential conductance similar to that shown in Fig. 2. This similarity is particularly pronounced when plotting the differential conductance (not shown) for $R$ of the same magnitude as $B$ in Fig. 2.

\section{Summary and conclusions}

Using the non-equilibrium Green function approach we have considered spin-polarized transport, associated with the Andreev reflection, through a single-level quantum dot coupled to one ferromagnetic and one superconducting leads. Features of the AR conductance due to the Zeeman spin-splitting of the dot energy level were analyzed in the context of similar effects induced in the AR linear conductance by the intradot spin-flip processes [3]. We have shown that the competition between broadening of the spin-split discrete dot's level and the Zeeman effect may lead to single or double resonances in the linear AR conductance. The latter is consistent with the previous result obtained for AR tunneling through a spin-split discrete level due to intradot spin-flip rotation [3]. We have also shown here that since the Zeeman spin splitting gives rise to a net spin polarization and spin accumulation in the dot, the AR double-peak conductance resonance may be strongly asymmetric. This feature is different from the corresponding one found for spin-flip rotation in the dot. When taking such processes into account one has to consider effects due to tunneling of a spin-polarized carrier into a superposition of spin up and spin down states in the dot. As a consequence, the double-peak of the linear conductance is symmetrical when the spin-flip scattering is strong enough.

In nonequilibrium situation, the numerical results reveal effects due to renormalization of the dot energy level when $\Gamma_{\mathrm{f} 0}<\Gamma_{\mathrm{s} 0}$. Such a renormalization is mainly due to the coupling between the dot and superconducting lead. For nonzero magnetic fields (or spin-flip processes), $B>0$ (or $R>0$ ), the resonance peaks in the differential conductance may become split into two peaks separated by the energy equal to $2 B$ (or $2 R$ ). With increasing QD-F coupling strength, $\Gamma_{\mathrm{f} 0}$, the effects due to renormalization of the dot discrete level become suppressed. However, at strong magnetic fields or for strong enough spin-flip coupling the double-peak feature still may be observed.

\section{Acknowledgments}

This work was supported by the Polish Ministry of Science and Higher Education as a research project in the years $2010-2013$.

\section{References}

[1] R.J. Soulen, Jr., J.M. Byers, M.S. Osofsky, B. Nadgorny, T. Ambrose, S.F. Cheng, P.R. Broussard, C.T. Tanaka, J. Nowak, J.S. Moodera, A. Barry, J.M.D. Coey, Science 282, 85 (1998).

[2] Y. Zhu, Q.-F. Sun, T.-H. Lin, Phys. Rev. B 65, 024516 (2001).

[3] X. Cao, Y. Shi, X. Song, S. Zhou, Phys. Rev. B 70, 235341 (2004).

[4] P. Zhang, Y.-X. Li, J. Phys., Condens. Matter 21, 095602 (2009).

[5] Z.Y. Zeng, F. Claro, Baowen Li, cond-mat/0110502.

[6] T. Domański, A. Donabidowicz, K.I. Wysokiński, Phys. Rev. B 78, 144515 (2008). 